\title{
Des mutations ponctuelles à l'origine des amyloses
}

Les protéines qui constituent les fibrilles amyloïdes ne sont pas homogènes. Depuis près de 20 ans, on a caractérisé plusieurs variétés : tout d'abord la protéine $\mathrm{AL}$, puis la protéine AA. De multiples autres protéines ont suivi, particulières à certains types cliniques d'amylose : protéine dérivée de la préalbumine dans les neuropathies amylö̈des familiales, dans la cardiomyopathie amyloïde familiale, et dans l'amylose cardiaque sénile, protéine dérivée de la calcitonine ou de la précalcitonine dans le carcinome médullaire de la thyroïde, protéine bêta dans la maladie d'Alzheimer, ou protéine dérivée de la bêta-2microglobuline dans l'amylose des malades dialysés (voir $\mathrm{m} / \mathrm{s} n^{\circ} 2$, vol. $2, p$. 106). La classification chimique et clinique des amyloses est détaillée dans un excellent éditorial de Husby et Sletten [I].

$\mathrm{La}$ protéine dite "gamma-trace variante " est le constituant principal des fibrilles amyloïdes présentes dans le tissu méningé des malades atteints d'hémorragie cérébrale héréditaire [2]. Cette maladie transmise selon le mode autosomique dominant, a été décrite en Islande dans huit familles, les accidents vasculaires cérébraux entraînant la mort avant l'âge de quarante ans; une autre famille a été récemment identifiée aux Pays-Bas. La protéine contient I Io résidus; elle est similaire à la protéine basique gamma-trace urinaire (ou cystatine C), mais elle ne commence qu'au I I $^{\mathrm{e}}$ résidu aminoterminal et comporte une substitution (glutamine remplaçant leucine) en position 58 (position 68 pour la molécule de protéine basique gamma-trace).

$\mathrm{La}$ protéine basique gamma-trace est présente chez l'homme dans le sérum et le liquide céphalorachidien (LCR), ainsi que dans certaines glandes endocrines. Sa concentration dans le LCR est normalement cinq fois plus élevée que dans le sérum, mais elle est très basse chez les malades atteints d'hémorragie cérébrale héréditaire. Il s'agirait d'un inhibiteur des cystéine-protéinases (d'où le terme " cystatine C "), enzymes qui regroupent, entre autres, les cathepsines humaines et la papaine. Le kininogène humain est également un inhibiteur de la papaine et de la cathepsine L. Il y a de nombreuses analogies de séquence entre ces divers inhibiteurs qui pourraient dériver d'un gène ancestral commun et posséder une structure exonique similaire.

Par comparaison avec la structure du gène des kininogènes humains, l'anomalie détectée au niveau de la protéine variante pourrait être une mutation ponctuelle d'un exon codant pour les acides aminés 56-93 (l'existence d'un tel exon vient d'être confirmée par l'étude du gène cloné codant pour la protéine basique gamma-trace [2]).

Cette mutation ponctuelle pourrait entraîner une anomalie du métabolisme de la molécule responsable de son dépôt et de son accumulation dans les tissus.

En utilisant une approche analogue, Wallace et coll. [3] ont caractérisé une nouvelle variante de la molécule de préalbumine constituant la substance amyloïde chez un malade nord-américain atteint de polyneuropathie amyloïde héréditaire. La substance amyloïde a été isolée du tissu cardiaque. La molécule variante contient de l'alanine en position 60 au lieu de la thréonine.
Le gène de la préalbumine a été récemment identifié et localisé sur le chromosome i 8 chez l'homme. Grâce à la connaissance de la séquence de l'ADNc de la préalbumine normale, les auteurs ont émis l'hypothèse d'une mutation ponctuelle en un point précis du codon. Cette hypothèse a été confirmée expérimentalement : la mutation supposée doit faire apparaitre un site supplémentaire de coupure par l'enzyme de restriction Pvu II. De fait, un tel site est observé au niveau du gène pathologique et non du gène normal lorsque des échantillons d'ADN de malades et de sujets témoins sont analysés par la technique du Southern blot (voir $m / s n^{\circ} 2$, vol. 2, p. 104).

Voilà donc de nouvelles variétés d'amylose héréditaire dont le constituant principal est caractérisé et dont la base moléculaire semble être une mutation ponctuelle d'un gène codant pour une protéine. Les anomalies du métabolisme des protéines variantes, produits des gènes mutés, seraient responsables de l'accumulation de la substance amyloïde. La détermination de l'anomalie génétique en cause permet d'envisager la détection précoce, éventuellement prénatale, des sujets atteints.

$$
\text { J.-P. G. et A. K. }
$$

I. Husby G, Sletten K. Chemical and clinical classification of amyloidosis 1985 . Scand $7 \mathrm{Immu}$ nol 1986; 23 : 253-65.

2. Ghiso J, Jensson O, Frangione B. Amyloid fibrils in hereditary cerebral hemorrhage with amyloidosis of icelandic type is a variant of gamma-trace basic protein (cystatin C). Proc Natl Acad Sci USA 1986; 83: 2974-8.

3. Wallace M R, Dwulet FE, Conneally P M, Benson M D. Biochemical and molecular genetic characterization of a new variant prealbumin associated with hcreditary amyloidosis. $\mathrm{f}$ Clin Invest $1986 ; 78:$ 6-12. 